\title{
ANÁLISIS DE LA EXACTITUD DEL MODELO DIGITAL DE TERRENO SRTM-30 PARA COLOMBIA USANDO DATOS IGAC E IDEAM
}

\author{
ANALYSIS OF THE ACCURACY OF THE DIGITAL MODEL OF AREA \\ SRTM-30 FOR COLOMBIA USING INFORMATION IGAC AND IDEAM
}

\author{
ANÁLISIS DE EXACTITUD DEL MODELO DE TERRENO DIGITAL PARA COLOMBIA
}

ACCURACY ANALYSIS OF THE DIGITAL TERRAIN MODEL FOR COLOMBIA

Carlos Capachero Martínez ${ }^{1}$, Pedro León García Reinoso ${ }^{2}$, Nelson Obregón Neira ${ }^{3}$
1. Ingeniero Ambiental y Sanitario de la Universidad Popular del César. E-mail: cacapacherom@unal.edu.co
2. Ingeniero Civil de la Universidad del Quindío. E-mail: pedro-garcia@javeriana.edu.co
3. Ingeniero Civil de la Universidad Francisco de Paula Santander. E-mail: nobregon@javeriana.edu.co
Recibido: 10 de Noviembre del 2014
Aceptado: 10 de Mayo del 2015
*Correspondencia: Doctorado en Ingeniería - Pontificia Universidad Javeriana Carrera 7 No. 42-27 Piso 7. Bogotá, Colombia. E-mail: pedro-garcia@javeriana.edu.co

\begin{abstract}
RESUMEN
Esta investigación se realizó con el propósito de estimar la exactitud vertical del MDT SRTM-30 para Colombia. El MDT SRTM-30 fue comparado con tres bases de datos que fueron suministradas por el IGAC y el IDEAM. Para el cálculo de la exactitud vertical se consideraron dos escenarios, a saber: un cálculo global y un cálculo discriminado por la pendiente del terreno. Además de calcular estadísticas como el error medio, la desviación estándar y el error cuadrático medio, también se realizaron las pruebas Koppe y NSSDA. Mientras que los valores obtenidos por el Test NSSDA mostraron dependencia con la pendiente del terreno, los valores del Test Koppe permanecieron relativamente estables en los dos escenarios de cálculo. Con un nivel de confianza del 95\% se determinó una exactitud vertical de $17 \mathrm{~m}$. Los resultados son importantes para entender el error asociado al MDT SRTM-30 y representan las características espaciales del error en función de la pendiente del terreno. Dado que el MDT SRTM-30 puede ser muy útil para adelantar estudios regionales, es posible adelantar otras investigaciones para mejorar la estimación de la exactitud vertical considerando aspectos como el tipo de cobertura del suelo y la morfología del terreno.
\end{abstract}

Palabras claves: Forma Terrestre, Procesamiento de datos, Análisis de datos, Sistema de información geográfica.

\begin{abstract}
This research was conducted with the purpose of estimating the vertical accuracy of the SRTM-30 MDT for Colombia. The MDT SRTM-30 was compared with three databases that were supplied by IGAC and IDEAM. For the calculation of the vertical accuracy were considered two scenarios, namely: general calculation and discriminated calculation that involved terrain slope. In addition to calculating statistics such as the mean error, the standard deviation and the root mean square, were also executed Test Koppe and Test NSSDA. While values obtained by Test NSSDA showed dependence with terrain slope, values from Test Koppe remained relatively stable in the two scenarios of calculation. With a confidence level of $95 \%$ was determined a vertical accuracy of $17.0 \mathrm{~m}$. Findings of the study are important to understand the error associated with SRTM-30 MDT and represents spatial characteristics of the error according to terrain slope. MDT SRTM-30 can be very useful for regional level study, further studies can be carried out to find the vertical accuracy considering aspects such as land cover and terrain morphology..
\end{abstract}

Keywords: Land Forms, Data processing, Data Analysis, Geographical information systems. 


\section{INTRODUCCIÓN}

En esta investigación aplicada se presta especial atención a la modelación del espacio geográfico a través de un Modelo Digital de Terreno (MDT) que es la representación estadística de la superficie continua del terreno por un gran número de puntos con coordenadas $\mathrm{X}, \mathrm{Y}, \mathrm{Z}$ conocidas en un campo de coordenadas arbitrario (1). Tanto la diversidad de fuentes como la facilidad de acceso a las mismas, ha permitido el desarrollo de estudios que considera el MDT como elemento fundamental para el estudio adecuado de algunos fenómenos naturales. Existen aplicaciones de MDT para el estudio de la erosión de suelos (2-5), el desarrollo de modelos hidrológicos (6), el estudio de glaciares (7), el estudio de volcanes (8), investigaciones en Geociencias (9-11), la delineación de áreas de inundación (12-13) y la evaluación del potencial hidroeléctrico en una cuenca hidrográfica (14-15), entre otros. Está ultima aplicación es la que motiva el desarrollo de esta investigación.

La potencia hidroeléctrica es la potencia obtenida de la energía del agua que cae. Las energías potencial y cinética del agua son utilizadas para generar energía eléctrica (16). La energía potencial es función de la diferencia de altura entre dos puntos a través de los cuales se presenta una caída de agua; lo que permite calcular la potencia hidroeléctrica a partir de la siguiente expresión,

\section{$P=\rho g h Q$}

Donde $P$ es la potencia (watts), $\rho$ es la densidad del agua $\left(\mathrm{kg} / \mathrm{m}^{3}\right)$, g es la aceleración debida a la gravedad $\left(\mathrm{m} / \mathrm{s}^{2}\right), h$ es la altura de la caída de agua (m) y $Q$ es el caudal (m3/s). En la Ecuación 1, las variables $h$ y $Q$ representan características propias del espacio geográfico. Mientras que $Q$ puede determinarse a partir de estudios hidrológicos, h se define a partir de la topografía que exhibe el terreno. La representación del terreno a través del MDT resulta útil para identificar el área y la red de drenaje de la cuenca hidrográfica en la que se calcula la potencia hidroeléctrica. La identificación adecuada de la cuenca hidrográfica y su red de drenaje depende de la resolución del MDT, a mayor resolución del MDT mejor podría ser la representación de la cuenca hidrográfica.

En Colombia, han sido utilizados MDT con pixeles de 90 metros para la delimitación de cuencas hidrográficas con aplicación en estudios hidrológicos (17). La identificación de la cuenca hidrográfica permite procesar tanto las variables físicas relacionadas con el potencial hidroenergético como describir el comportamiento de las aguas superficiales a lo largo de la red de drenaje que ha sido trazada en el MDT. En esta investigación, se determinó la exactitud en la distancia vertical del Modelo Digital de Terreno SRTM-30 generado por la Shuttle Radar Topography Mission para decidir el uso potencial que tendría este producto en aplicaciones prácticas como la identificación de la red de drenaje en el territorio continental colombiano, el cálculo de parámetros morfométricos empleados en modelos hidrológicos y la estimación de la incertidumbre generada en el cálculo de la potencia hidroeléctrica.

\section{MATERIALES Y MÉTODOS}

Este trabajo se desarrolló como una investigación aplicada en la que se utilizó el software ArcMap 10.1 $1^{1}$ y R (18) para el procesamiento y análisis de la información. En la Figura 1 se ilustra la metodología empleada. El primer paso consistió en la construcción del mosaico de 601 segmentos del MDT para Colombia referenciados en el Datum Magna Transversa de Mercator con un tamaño de celda de $30 \mathrm{~m}$ y una profundidad de pixel de 16 Bit. En la elaboración del mosaico se utilizó información suministrada por el IGAC con referencia al MDT SRTM-30 en formato GRID, la cual se corresponde con cartografía básica a escala 1:100.000 en formato GDB. Posteriormente se generó un mapa de pendientes para analizar el efecto de la pendiente del terreno sobre el error del MDT. Para simplificar el tratamiento de la información raster y vectorial, fueron definidas cuatro categorías de pendiente, las cuales se adaptaron de la clasificación convencional del IGAC que se presenta en la Tabla 1. Bajo esta reclasificación se generó el mapa de pendientes de Colombia que se muestra en la Figura 2.

Los datos y mapas presentados en este artículo fueron procesados usando software ArcGIS® by Esri. ArcGIS® es propiedad intelectual de Esri y aqui fue usado bajo licencia. Copyright (C) Esri. Todos los derechos reservados. Para más información, por favor visitar www.esri.com 


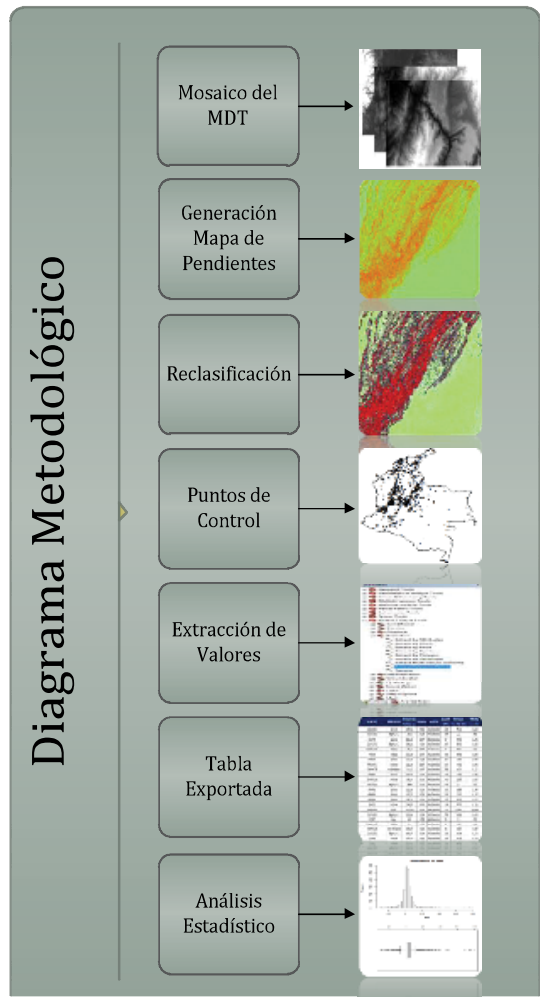

Figura 1. Metodología del Estudio

Tabla 1. Clasificación de pendientes para Colombia.

\begin{tabular}{cccc}
\hline & & PENDIENTE & \\
Unidad & $\%$ & $\%$ Área Nacional & Clasificación \\
\hline 1 & $<12$ & $67 \%$ & Suave \\
2 & $12-25$ & $13 \%$ & Moderada \\
3 & $25-50$ & $12 \%$ & Abrupta \\
4 & $>50$ & $7 \%$ & Escarpada \\
\hline
\end{tabular}

Generalmente el análisis del error en la componente vertical de los MDT se hace calculando el Error Medio Cuadrático a partir de la elevación estimada en el modelo y la resultante de una fuente de mayor precisión (20). Para el caso de Colombia, se conformaron tres bases de datos con la información secundaria oficial reportada en la red Geodésica Nacional del IGAC (21) y en la red de Estaciones Hidrometeorológicas IDEAM (22), ambas fuentes en tipo punto y formato Shapefile. La distribución de esta información puede observarse en la Figura 3.

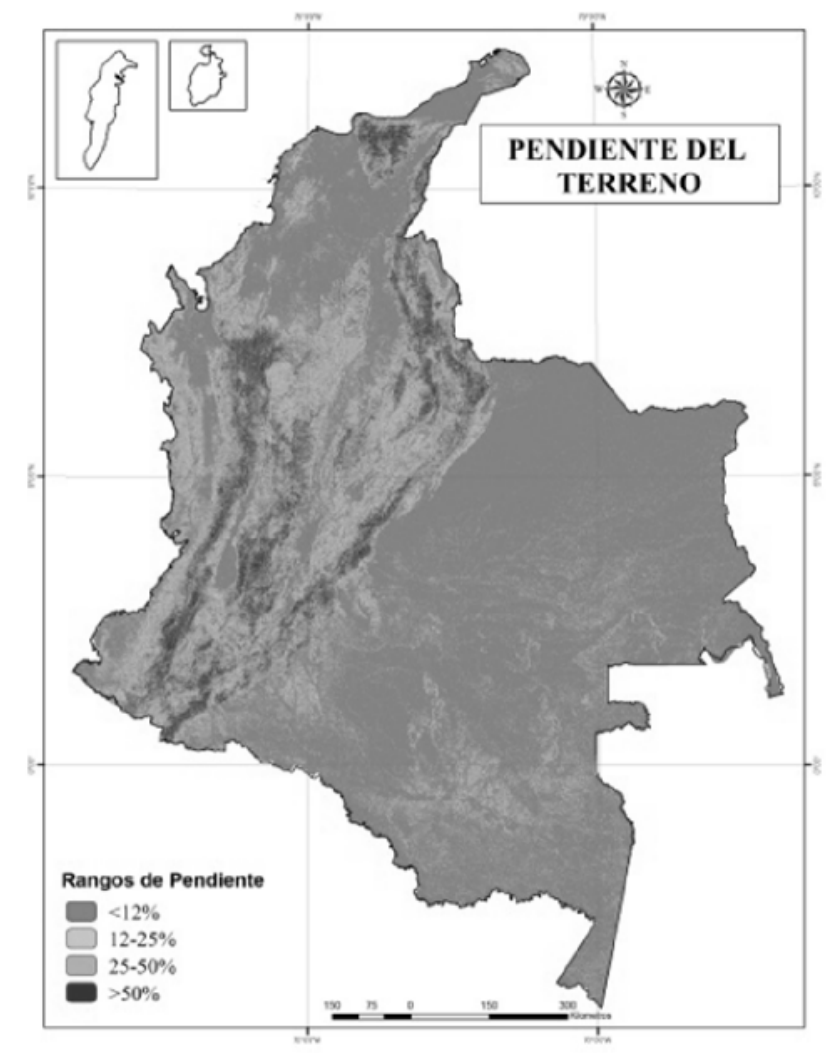

Figura 2. Mapa de pendientes de Colombia (19).

Continuando con la propuesta metodológica ilustrada en la Figura 1, los puntos de control se obtuvieron extrayendo la información de la altura contenida en las celdas del MDT y los valores de pendiente del terreno, estos últimos tanto en porcentaje como en grados. Estos valores fueron procesados haciendo uso de un algoritmo de interpolación bilineal que considera información relevante de las cuatro celdas vecinas, almacenando el nuevo dato en un archivo tipo punto. Con la información cartográfica configurada, se procedió al cálculo de la diferencia de altura entre el MDT y la red Geométrica del IGAC. Este procedimiento se repitió con las bases de datos construidas a partir de datos GPS, métodos trigonométricos y la información de la red de estaciones del IDEAM. De esta forma se elaboraron tres conjuntos de datos sobre los cuales se realizó el cálculo del error medio, la desviación estándar, el error medio cuadrático, las pruebas de Koppe y National Standard for Spatial Data Accuracy (NSSDA) (24). El cálculo del error medio en estos conjuntos de datos tiene como interés princi- 

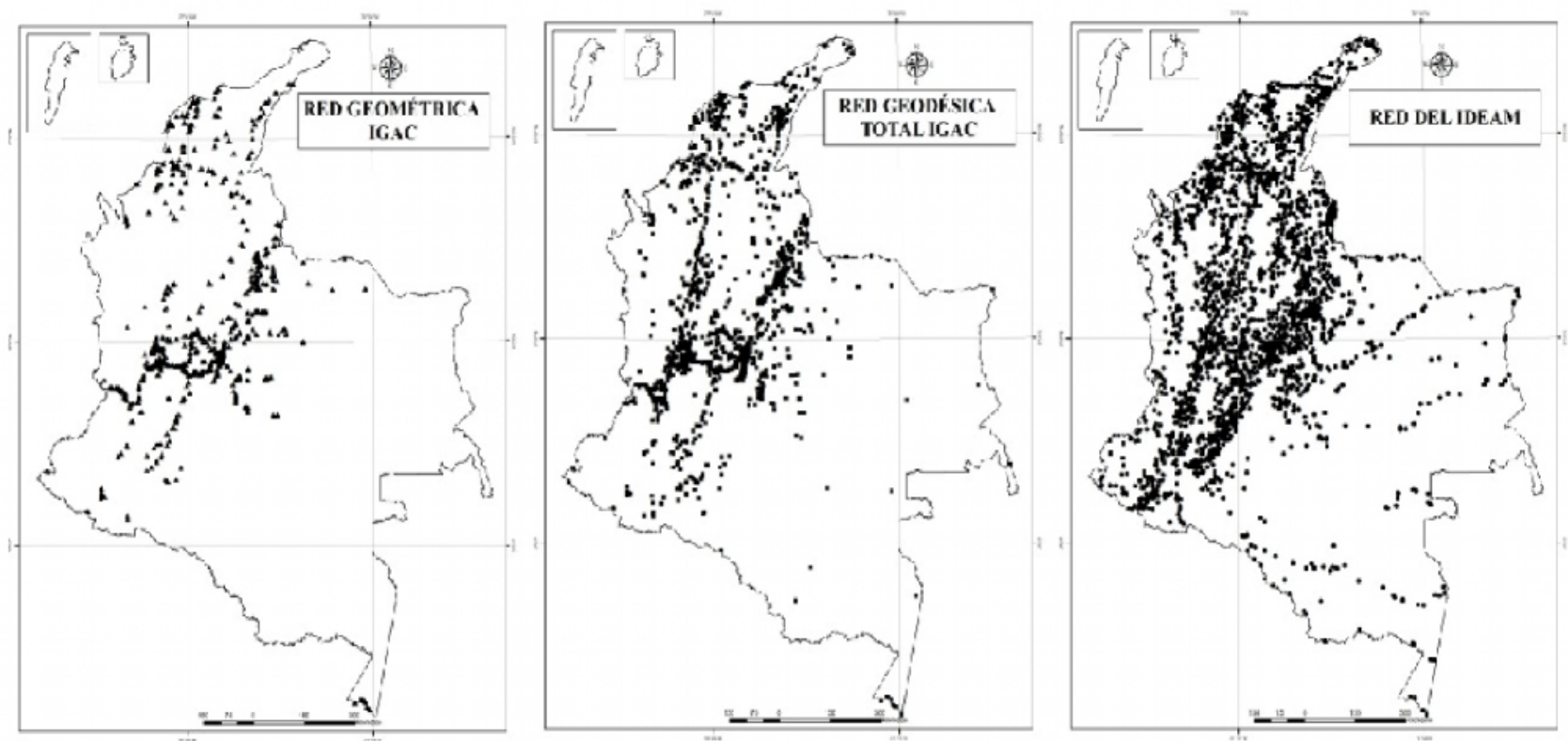

Figura 3. Distribución datos IGAC e IDEAM en el territorio Colombiano (23).

pal comprobar la aleatoriedad de las desviaciones del modelo, de esta manera cuando los errores presentan un patrón que siguen un arreglo y pueden producir un sesgo en el producto final se les conoce como sistemático, si por el contrario son aleatorios y se presentan en diferentes zonas del MDT sin un patrón constante se le denomina error no sistemático (25).

Otras pruebas como la EMAS/ASPRS (Engineering Map Accuracy Standard /American Society of Photogrammetry and Remote Sensing) y NMAS (National Map Accuracy Standard) no se realizaron bien sea por el número de puntos de control disponibles o porque generalmente se emplean para el control de calidad posicional cartográfica, aspecto que supera el propósito de este trabajo.

\section{RESULTADOS}

En la Tabla 2 se presenta un resumen del análisis estadístico que se realizó para cada uno de los tres conjuntos de datos conformados para analizar la exactitud del MDT SRTM-30. La segunda columna relaciona los resultados del análisis para los 1262 puntos que conforman las Alturas Geométricas IGAC, la tercera columna presenta los resultados del análisis de los 3045 puntos que conforman las Alturas Geométricas y los datos GPS IGAC, mientras que en la cuarta columna se relacionan la altura que reporta el IDEAM para las estaciones que conforman su red de medición hidrometeorológica. En una revisión preliminar global se observa que el error medio tiende a cero, un comportamiento que sugiere la posible existencia de un error sistemático del MDT SRTM-30. La división del país en rangos de pendiente permitirá estudiar con mayor detalle el comportamiento que podría exhibir este error.

Tabla 2. Análisis estadístico entre el MDT SRTM-30 y datos IGAC e IDEAM

\begin{tabular}{lccc}
\hline Estadísticos & $\begin{array}{c}\text { Alturas } \\
\text { Geomé- } \\
\text { tricas } \\
\text { IGAC }\end{array}$ & $\begin{array}{c}\text { Datos } \\
\text { Totales } \\
\text { IGAC }\end{array}$ & $\begin{array}{c}\text { Alturas } \\
\text { de Es- } \\
\text { taciones } \\
\text { IDEAM }\end{array}$ \\
\hline Error Mínimo (m) & -29.51 & -62.50 & -6914.00 \\
Error Máximo (m) & 79.62 & 79.62 & 2328.30 \\
Número de Puntos (m) & 1262 & 3045 & 4349 \\
Error Medio (m) & 2.92 & 1.24 & 4.76 \\
Error Cuadrático Medio (m) & 9.51 & 10.77 & 229.84 \\
Desviación Estándar (m) & 9.05 & 10.70 & 229.79 \\
Sesgo & 2.5 & 0.97 & -7.25 \\
Curtosis & 21.81 & 13.48 & 218.49 \\
Test NSSDA (m) & 18.64 & 21.10 & 450.48 \\
Test Koppe (m) & 16.93 & -3.88 & 198.53 \\
\hline
\end{tabular}

Las estadísticas que se reportan en la Tabla 2 son indicativas de que el conjunto de datos que mejor describe el MDT SRTM-30 es el de Alturas Geométricas IGAC, que pese a que indica un incremento en el error medio al compararlo con el conjunto Datos 
Totales IGAC, muestra una reducción del error cuadrático medio, el error mínimo y la desviación estándar al compararlo con el mismo conjunto de datos. Puesto que los valores reportados por el análisis estadístico para el conjunto de datos Altura de Estaciones IDEAM no son comparables con los valores reportados por los conjuntos de datos IGAC es posible asumir que la fuente de mayor precisión para comparar el MDT SRTM-30 es el conjunto de datos de Alturas Geométricas IGAC.

Con respecto al conjunto de datos Alturas Geométricas IGAC, la exactitud en la vertical con un nivel de confianza del $95 \%$ es de $18.64 \mathrm{~m}$ según el Test NSSDA y se reduce a $16.93 \mathrm{~m}$ de acuerdo con el resultado obtenido para el Test de Koppe. Los coeficientes de Curtosis y Sesgo indican para el error una distribución leptocúrtica y asimetría positiva, revelando una agrupación significativa alrededor de cero con un rápido decaimiento hacia valores extremos (Figura 4), en los cuales se muestra claramente la tendencia que tiene la superficie modelada por los datos MDT SRTM-30 a subestimar los puntos que conforman el conjunto de datos Alturas Geométricas IGAC, por ello es posible identificar un número mayor de puntos por debajo de la superficie modelada (Figura 5).
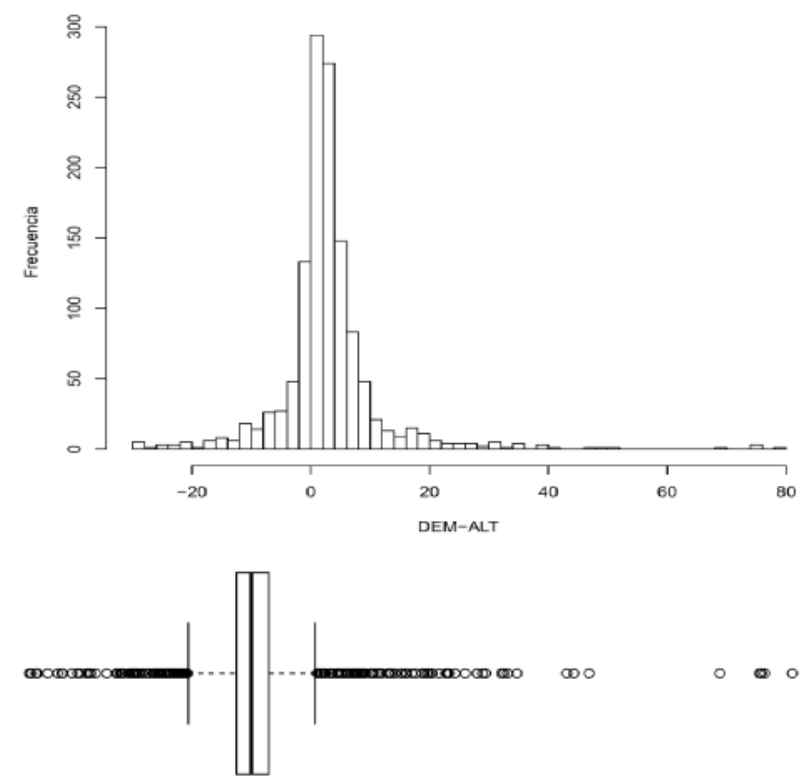

Figura 4. Histograma de las diferencias entre el MDT SRTM-30 y el conjunto de datos Alturas Geométricas IGAC

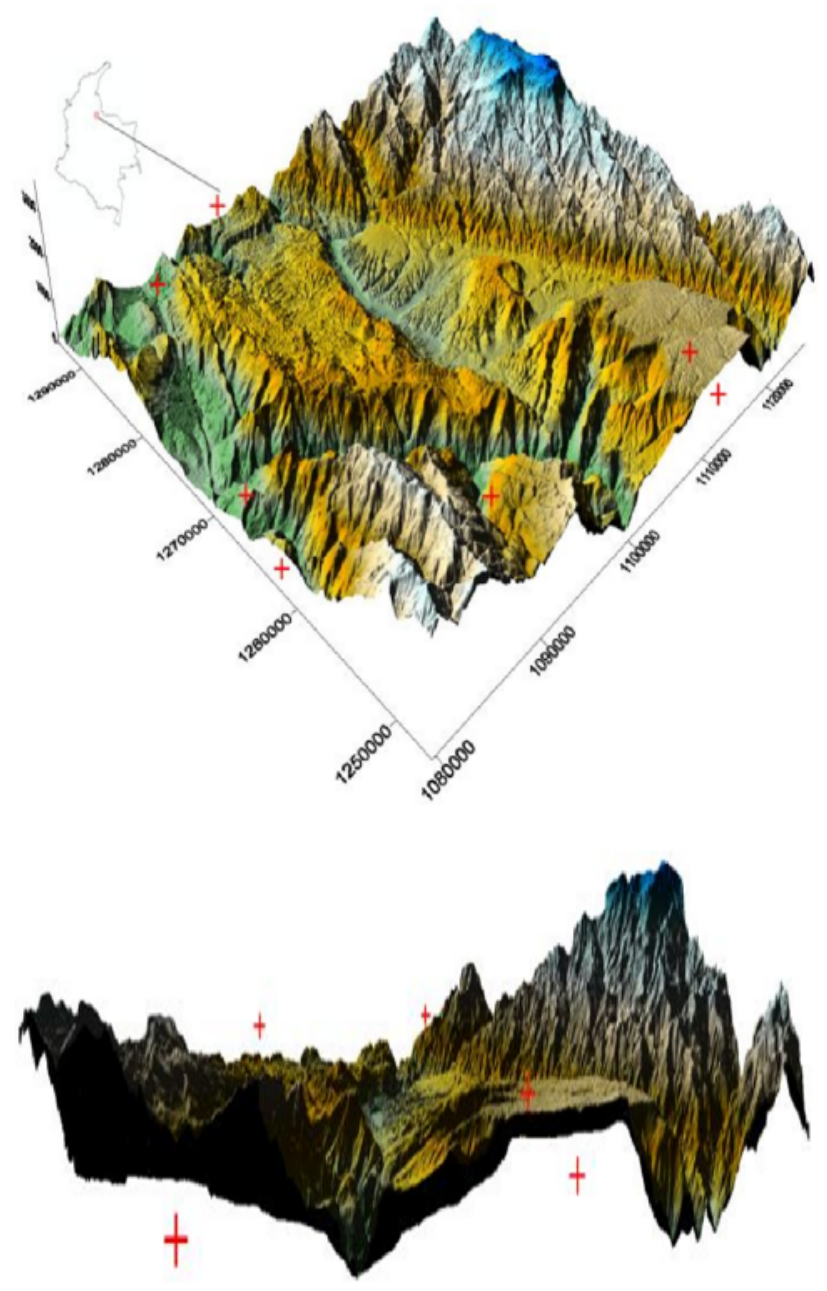

Figura 5. MDT SRTM-30 con relación a puntos subestimados y sobreestimados

Tabla 3. Análisis estadístico entre el MDT SRTM-30 y los datos Alturas Geométricas IGAC para distintos rangos de pendiente en Colombia

\begin{tabular}{lcccc}
\hline \multicolumn{1}{c}{ Estadísticos } & $<\mathbf{1 2 \%}$ & $\mathbf{1 2 - 2 5 \%}$ & $\mathbf{2 5 - 5 0 \%}$ & $\mathbf{> 5 0 \%}$ \\
\hline Error Mínimo (m) & -29.50 & -25.45 & -24.72 & -29.51 \\
Error Máximo (m) & 27.41 & 75.21 & 69.26 & 79.62 \\
Número de Puntos & 991 & 162 & 87 & 22 \\
Error Medio (m) & 2.04 & 4.47 & 8.04 & 10.96 \\
$\begin{array}{l}\text { Error Cuadrático } \\
\text { Medio (m) }\end{array}$ & 4.81 & 14.65 & 18.54 & 34.80 \\
$\begin{array}{l}\text { Desviación Estándar } \\
\text { (m) }\end{array}$ & 4.35 & 13.95 & 16.70 & 33.03 \\
Test NSSDA (m) & 9.42 & 28.72 & 36.33 & 68.22 \\
Test Koppe (m) & 16.92 & 16.95 & 16.99 & 17.04 \\
\hline
\end{tabular}


Los resultados de la evaluación de la exactitud vertical del MDT SRTM-30 con respecto al conjunto de datos Alturas Geométricas IGAC considerando la pendiente del terreno se reportan en la Tabla 3. Para el 67\% del territorio colombiano continental, reclasificado con una pendiente suave, se obtuvo un error medio de $2.04 \mathrm{~m}$, el cual crece con el aumento de la pendiente hasta alcanzar un valor de $10.96 \mathrm{~m}$ para un terreno escarpado. El error cuadrático medio exhibe un comportamiento similar al error medio, variando entre $4.81 \mathrm{~m}$ para zonas con pendiente suave hasta $34.80 \mathrm{~m}$ para zonas con pendiente escarpada. Las regiones de pendiente suave se corresponden con zonas de baja de información como son Orinoquía y Amazonía, donde existe un número muy reducido de datos con respecto a otras zonas del país, lo que podría explicar el incremento de la desviación estándar con el aumento de la pendiente. Mientras que el Test NSSDA es notablemente sensible al incremento de la pendiente, el Test Koppe produce valores relativamente estables para los diferentes rangos de pendiente, reportando un valor aproximado de $17.0 \mathrm{~m}$.

\section{DISCUSIÓN}

Los resultados muestran que el mayor error se encuentra en terrenos de alta pendiente mientras que en zonas de pendiente baja sucede lo contrario. Este comportamiento ha sido relacionado en otras investigaciones, en las cuales se concluyó que la exactitud vertical del MDT SRTM se encuentra influenciada por la pendiente del terreno. Una de ellas analizó la exactitud vertical del MDT SRTM contra datos recolectados con GPS en dos zonas con topografía diferente (26), los datos fueron divididos en dos conjuntos: menores a $10^{\circ}$ y mayores a $10^{\circ}$, reportando el mayor error para zonas con pendientes superiores a $10^{\circ}$. En otra investigación se estudió el efecto del MDT SRTM sobre el desempeño de un modelo hidrológico para diferentes partes de la cuenca (6), identificando el menor error en las zonas planas y errores superiores a los $30 \mathrm{~m}$ en zonas con pendiente cercana a los $50^{\circ}$. En esta investigación, los errores encontrados cubren un rango que va de $2.04 \mathrm{~m}$ en terreno con pendiente suave, hasta $10.96 \mathrm{~m}$ en terreno con pendiente escarpada. El error medio general es de $2.92 \mathrm{~m}$.

Para el conjunto de datos Alturas Geométricas IGAC, el Test NSSDA produce un valor de exactitud vertical de $18.64 \mathrm{~m}$ mientras que el Test Koppe genera un valor de $16.93 \mathrm{~m}$. Pero aunque las dos pruebas generan valores similares para todo el conjunto de datos, el
Test NSSDA resulta sensible a la pendiente del terreno ya que reporta $9.42 \mathrm{~m}$ en pendientes menores al $12 \%$ y $68.22 \mathrm{~m}$ en pendientes mayores al $50 \%$. En ambos casos la tendencia es similar, evidenciando un incremento del error del modelo en la medida que la pendiente del terreno es mayor. Esta afirmación ha sido motivo de numerosas investigaciones, en las cuales se sugiere que la relación entre el error del modelo y la pendiente del terreno se encuentra en función de la rugosidad de la superficie $(6,27-29)$ puesto que cuando el terreno presenta topografía escarpada, los sensores no logran captar la complejidad del terreno lo que genera perdida en los detalles menores del paisaje (30). Situación contraria sucede en terrenos con pendiente suave, donde las alturas observadas son relativamente constantes y los gradientes de pendiente no resultan ser tan pronunciados como en terrenos montañosos como los predominantes en el territorio continental colombiano. Otros autores atribuyen estos errores no solo a la topografía, sino también a las condiciones meteorológicas que pueden alterar los tiempos de trayecto de la radiación emitida por los sensores y los cambios de la vegetación en la superficie (31), a la sombra de radar $(6,29)$ o a la resolución espacial del modelo, inversamente proporcional a la fidelidad con que se representa la topografía (32).

Dado que el error medio indica un error sistemático y considerando que son válidos, para el conjunto de datos Alturas Geométricas IGAC, los resultados del Test Koppe se puede establecer un valor aproximado de exactitud vertical de $17.0 \mathrm{~m}$ en el territorio continental colombiano. Este valor es consecuente con los valores que la NASA obtuvo al comparar datos MDT SRTM con datos DTED Level 2 para Suramérica (33): una diferencia de altura promedio de $11.36 \mathrm{~m}$ con una desviación estándar de $4.60 \mathrm{~m}$.

Resulta claro que los resultados de la evaluación de la exactitud dependen del número de datos por una unidad espacial de los datos MDT SRTM-30. Cuantos más datos estén disponibles, más cercano a la realidad será el cálculo de la exactitud vertical. Sin embargo, la materialización de puntos geodésicos para las Estaciones IDEAM demanda una inversión de recursos adicionales que no estaban incluidos dentro del alcance de esta investigación. Por ello es necesario mencionar, que aunque se analizó un amplio conjunto de datos, éstos no resultan suficientes para cubrir todo el territorio continental colombiano. En algunas áreas no se realizaron cálculos, ya sea porque no cuenta con 
información, o porque se presentaban errores atípicos que reflejaban la inconsistencia de los datos. También se debe acotar que los datos analizados corresponden con la información institucional disponible y que éstos fueron recolectados siguiendo técnicas y metodologías diferentes. En este sentido y con el ánimo de promover la integración institucional, es recomendable que fuentes de información como la Red Geodésica Nacional del IGAC, la Red de Estaciones Hidrometeorológicas del IDEAM y la Red Sismológica del Servicio Geológico Colombiano, generen acuerdos alrededor de metodologías que permitan definir de forma unificada la precisión posicional y altimétrica de las estaciones que correspondan a cada institución. Los resultados son importantes para entender el error asociado al MDT SRTM-30, representan las características espaciales del error en función de la pendiente del terreno. Dado que el MDT SRTM-30 puede ser muy útil para adelantar estudios regionales en diferentes tópicos, es posible adelantar otras investigaciones para mejorar la estimación de la exactitud vertical considerando aspectos como el tipo de cobertura del suelo y la morfología del terreno, incluyendo datos recolectados de forma sistemática y ajustada a un estándar nacional de carácter institucional. Comparando la exactitud vertical que ha sido determinada en esta investigación contra la reportada en la literatura, se puede recomendar el uso del MDT SRTM-30 para reproducir la red de drenaje en escalas iguales o superiores a 1:100.000.

\section{AGRADECIMIENTOS}

Este trabajo fue realizado con el apoyo técnico y logístico del Instituto Geofísico de la Pontificia Universidad Javeriana y el Grupo de Investigación Ciencia e Ingeniería del Agua, en el marco del proyecto "Evaluación del Potencial Hidroenergético de Colombia" que fue financiado por la Convocatoria 558 de Colciencias. El grupo de trabajo de la Pontificia Universidad Javeriana agradece la colaboración de la Unidad de Planeación Minero Energética, la contribución del Instituto Geográfico Agustín Codazzi por el suministró del MDT SRTM-30 y la información vectorial de la red geodésica nacional, y la cooperación del Instituto de Hidrología Meteorología y Estudios Ambientales IDEAM que suministró la información descriptiva de las estaciones hidrometeorológicas.

\section{BIBLIOGRAFIA}

1. Li Z., Zhu C., Gold C. Digital Terrain Modeling: Principles and Methodology. Florida: CRC Press; 2010. pág. 7.

2. Rennó, C. D., Nobre, A. D., Cuartas, L. A., Soares, J. V., Hodnett, M. G., Tomasella, J., Waterloo, M. J. HAND, a new terrain descriptor using SRTM-DEM: Mapping terra-firme rainforest environments in Amazonia. Remote Sensing of Environment, 2008; 112 (9): 3469-3481.

3. Guth, P. L. Geomorphometry from SRTM : Comparison to NED. Photogrammetric Engineering \& Remote Sensing, 2006, 72 (3): 269-277.

4. Siart, C., Bubenzer, O., Eitel, B. Combining digital elevation data (SRTM/ASTER), high resolution satellite imagery (Quickbird) and GIS for geomorphological mapping: A multi-component case study on Mediterranean karst in Central Crete. Geomorphology, 2009, 112 (2): 106-121.

5. Kinsey-Henderson, A. E., Wilkinson, S. N. Evaluating Shuttle radar and interpolated DEM's for slope gradient and soil erosion estimation in low relief terrain. Environmental Modelling \& Software, 2013, 40: 128-139.

6. Ludwig, R., Schneider, P. Validation of digital elevation models from SRTM X-SAR for applications in hydrologic modeling. ISPRS Journal of Photogrammetry and Remote Sensing, 2006, 60 (5): 339-358.

7. Racoviteanu, A. E., Manley, W. F., Arnaud, Y., Williams, M. W. Evaluating digital elevation models for glaciologic applications: An example from Nevado Coropuna, Peruvian Andes. Global and Planetary Change, 2007, 59 (4): 110-125. 
8. Grosse, P., Van Wyk de Vries, B., Euillades, P. a., Kervyn, M., Petrinovic, I. Systematic morphometric characterization of volcanic edifices using digital elevation models. Geomorphology, 2012, 136 (1): 114-131.

9. Mantelli, L. R., Rossetti, D. D. F., Albuquerque, P. G., Valeriano, M. D. M. Applying SRTM digital elevation model to unravel Quaternary drainage in forested areas of Northeastern Amazonia. Computers \& Geosciences, 2009, 35 (12): 2331-2337.

10. Hayakawa, E. H., Rossetti, D. F., Valeriano, M. M. Applying DEM-SRTM for reconstructing a late Quaternary paleodrainage in Amazonia. Earth and Planetary Science Letters, 2010, 297 (2): 262-270.

11. Masoud, A. a., Koike, K. Auto-detection and integration of tectonically significant lineaments from SRTM DEM and remotely-sensed geophysical data. ISPRS Journal of Photogrammetry and Remote Sensing, 2011, 66 (6): 818-832.

12. Pan, F., Liao, J., Li, X., Guo, H. Application of the inundation area-lake level rating curves constructed from the SRTM DEM to retrieving lake levels from satellite measured inundation areas. Computers \& Geosciences, 2013, 52: 168-176.

13. Burgos, V. Evaluación de ASTER GDEM y SRTM-C / X para modelación hidráulica de la rotura de presa El Carrizal, Mendoza. Instituto Nacional del Agua - Centro Regional Andino. 2012.

14. Venkatesan, V., Balamurugan, R., Krishnaveni, M. Establishing Water Surface Area-Storage Capacity Relationship of Small Tanks Using SRTM and GPS. Energy Procedia, 2012, 16: 11671173.

15. Fitzgerald, N., Lacal, R., McKeogh, E., Leahy, P. A GIS-based model to calculate the potential for transforming conventional hydropower schemes and non- hydro reservoirs to pumped hydropower schemes. Energy, 2012, 41 (1): 483-490.

16. Enríquez G. Tecnologías de generación de energía eléctrica. México: Limusa; 2009.

17. IDEAM. Estudio Nacional del Agua 2010. Bogotá: Instituto de Hidrología, Meteorología y Estudios Ambientales; 2010. pág. 62.

18. R Development Core Team. R: A language and environment for statistical computing. Vienna: R Foundation for Statistical Computing: 2008.

19. Capachero C.A. Mapa de pendientes de Colombia [Figura]. Escala no vista. "Proyecto de Investigación: Evaluación del Potencial Hidroenergético de Colombia" 2013. Software: ArcGIS [software GIS]. Versión 10.0. Redlands, CA: Environmental Systems Research Institute, Inc., 2010.

20. Pérez A., François J. Evaluación de los errores de modelos digitales de elevación obtenidos por cuatro métodos de interpolación. En Investigaciones Geográficas, Boletín del Instituto de Geografía, UNAM, 2009; (69), 53-67.

21. Instituto Geográfico Agustín Codazzi. [SWAMI 3.0]. Bogotá: IGAC; [06/09/2013]. http:// srvmetadata.igac.gov.co/swami/

22. Instituto de Hidrología, Meteorología y Estudios Ambientales de Colombia. [Catálogo Nacional de Estaciones del IDEAM]. Bogotá: IDEAM; [08/09/2013]. http://institucional.ideam.gov.co/ jsp/index.jsf

23. Capachero C.A. Distribución de los datos del IGAC e IDEAM en el territorio Colombiano [Figura]. Escala no vista. "Proyecto de Investigación: Evaluación del Potencial Hidroenergético de Colombia” 2013. Software: ArcGIS [software GIS]. Versión 10.0. Redlands, CA: Environmental Systems Research Institute, Inc., 2010.

24. Atkinson A.D.J., García J.L., Ariza F.J. Los diferentes test para el control de calidad posicional en cartografía. Cáceres: Universidad de Extremadura. Departamento de Expresión Gráfica, 2001. pág. 3 .

25. Wechsler, S. P., Kroll, C. N. Quantifying DEM Uncertainty and its Effect on Topographic Parameters, 2006, 72 (9): 1081-1090. 
26. Gorokhovich Y., Voustianiouk A. Accuracy assessment of the processed SRTM-based elevation data by CGIAR using field data from USA and Thailand and its relation to the terrain characteristics. En Remote Sensing of Environment, 2006; (104): 409-415.

27. Mukherjee S., Joshi P.K., Mukherjee S., Ghosh A., Garg R.D., Mukhopadhyay A. Evaluation of vertical accuracy of open source Digital Elevation Model (DEM). International Journal of Applied Earth Observation and Geoinformation, 2013, 21: 205-217.

28. Hengl T., Reuter H.I. Geomorphometry: Concepts, Software, Applications. The Netherlands: Elsevier; 2009.

29. Toutin, T. Erratum to "Impact of terrain slope and aspect on radargrammetric DEM accuracy" [ISPRS J. Photogramm. Remote Sens. 57 (2002) 228-240]. ISPRS Journal of Photogrammetry \& Remote Sensing, 2003, 58: 124- 125.

30. Fallas, J. Modelos digitales de elevación: Teoría, métodos de interpolación y aplicaciones. Costa Rica: Universidad Nacional, Escuela de Ciencias Ambientales; 2007.

31. Fernádez T., Irigaray C., El Hamdouni R., Chacón J. Correlation between natural slope angle and rock mass strength rating in the Betic Cordillera, Granada, Spain. Bulletin of Engineering Geology and The Environment, 2008, 67:153-164.

32. Lencinas J.D., Díaz G.M. Corrección geométrica de datos satelitales QuickBird, incidencia de los modelos digitales de elevación SRTM-C/X y ASTER GDEM. GeoFocus, 2011, 11: 431-454. .

33. NASA. An assessment of the SRTM topographic products. Technical Report JPL D-31639. Pasadena: Jet Propulsion Laboratory; 2005. pág. 34. 